\title{
Effects of Gravity on Gastric Emptying, Intestinal Transit, and Drug Absorption
}

\author{
Gordon L. Amidon, PhD, Gary A. DeBrincat, MS, and Naji Najib, PhD
}

\begin{abstract}
The effects of microgravity on the physiologic response of the human body, the physical properties of gastrointestinal contents, and the influence these responses have on drug absorption are becoming more and more critical as the duration of humans in the hostile space environment dramatically increases. In this environment, some conventional oral dosage forms may be severely limited as an effective drug regimen. To understand the effects of microgravity, one must first understand the basic forces acting on a particle moving through a walled-tube such as the small intestine: gravity $\left(F_{G}\right)$, buoyancy $\left(F_{B}\right)$, and drag $\left(F_{\mathrm{D}}\right)$. These forces can be combined and rearranged into a dimensionless ratio of gravitational forces to viscous forces. This is the most important dimensionless group influencing the motion of a particle relative to the fluid. Gastric emptying is highly influenced by several factors: volume, calories, exercise, size, density, temperature, viscosity, osmolality as well as those factors associated with physiologic responses: splanchnic blood flow, body position, and electrolyte balance. This array of factors can lead to variability in drug plasma levels. In the absence of gravity, the factors of size and density would appear to be most directly altered due to their dependence on the force of gravity. Intestinal transit rate in a gravity environment is highly dependent on the motility state of the GI tract either fasted or fed partly due to the higher viscosities of chyme in the fed state. In space, the absence of gravity may tend to increase the transit rate along the small intestine by decreasing the dimensionless ratio of gravitational forces to viscous forces. In zero gravity, therefore, these alterations in GI emptying and intestinal transit rate could lead to erratic plasma levels and inefficient absorption.
\end{abstract}

$\mathbf{S}^{\mathrm{p}}$ paceflight is associated with numerous changes Sthat may influence human biological systems. The most remarkable among these is the relative absence of gravity. This significant change subjects astronauts to a unique environmental situation to which their bodies are not well adapted.

With the increasing length of the space shuttle flights, the proposed establishment of a permanent space station, and a possible manned flight to the planet Mars, increasing duration of humans in space further substantiates the need for developing a drug therapy consistent with a microgravity environment. This therapy is essential to future space exploration since increasing exposure to microgravity results in an increasing incidence of situations in which the use of medications might be indicated. Hence, knowledge of the pharmacokinetics and phar-

From the College of Pharmacy, The University of Michigan, Ann Arbor, Michigan. Address for reprints: Gordon L. Amidon, PhD, The College of Pharmacy, The University of Michigan, Ann Arbor, MI 48109-1065. macodynamics of drugs in normal situations may not prevail in conditions of microgravity and establishment of a research program aimed at elucidating and characterizing the pharmacokinetics and pharmacodynamics of drugs that might be used in space is of prime importance.

In many of the space shuttle missions to date, the astronauts were highly susceptible to space motion sickness (SMS) characterized by nausea and vomiting during the first few days of the mission. In addition, scopolamine/detroamphetamine tablets in a conventional dosage regimen have been used to alleviate these symptoms, but the inflight salivary concentration-time curves of these drugs are erratic and possess a high intersubject and intrasubject variability when compared with preflight data. ${ }^{1}$ These findings can possibly be attributed to erratic gastric emptying, intestinal transit, and drug absorption since each of these parameters can change in a microgravity environment. Some considerations related to each of these possibilities are given below. 


\section{GASTRIC EMPTYING AND INTESTINAL TRANSIT}

Gastric emptying, i.e., the rate at which a substance leaves the stomach and enters the small intestine (the primary absorptive site for most oral drugs) and intestinal transit, i.e., the rate at which particles leaving the stomach traverse the intestine are dependent on two main groups of factors:

1. Factors that are related to the physical properties of the solid and liquid contents of the stomach and intestine

2. Factors that are concerned with the physiologic, hormonal, and enzymatic changes that might occur directly in the stomach and intestine or to other body organs

\section{Physical Properties}

Oral drug and nutrient bioavailability requires proper gastric emptying and adequate absorption while traversing the small intestine. The physical variables that influence gastric emptying and intestinal transit are best discussed on a fluid mechanical basis. First, consider a spherical particle in a fluidfilled horizontal smooth tube. There are three major forces acting on the particle: gravity $\left(\mathrm{F}_{\mathrm{G}}\right)$, buoyancy $\left(F_{B}\right)$, and drag $\left(F_{D}\right)$ (Figure). These three forces are given by the following equations: ${ }^{2}$

$$
\begin{aligned}
& \mathrm{F}_{\mathrm{G}}=\frac{\pi \mathrm{d}_{\mathrm{p}}^{3}}{6} \mathrm{~g} \rho_{\mathrm{p}} \\
& \mathrm{F}_{\mathrm{B}}=\frac{\pi \mathrm{d}_{\mathrm{p}}^{3}}{6} \mathrm{~g} \rho_{\mathrm{f}} \\
& \mathrm{F}_{\mathrm{D}}=\frac{\pi}{8} \rho_{\mathrm{f}}\langle\mathrm{v}\rangle^{2} \mathrm{dp}^{2} \mathrm{C}_{\mathrm{D}}
\end{aligned}
$$

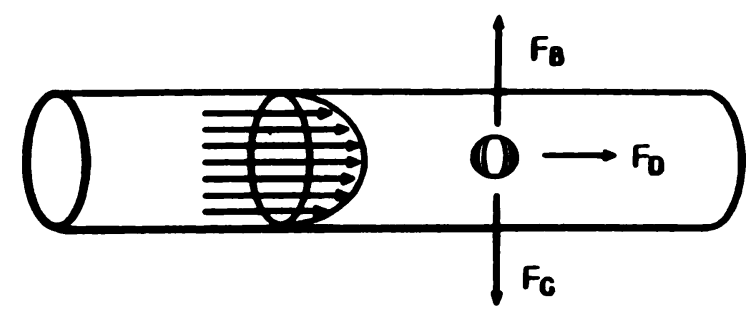

Figure. Forces acting on a particle within a fluid-filled smooth tube under flow conditions. where $\rho_{\mathrm{p}}$ is the particle density, $\rho_{\mathrm{f}}$ is the fluid density, $d_{p}$ is the particle diameter, $<v>$ is the average velocity, and $C_{D}$ is the drag coefficient.

It is convenient to define another term $\mathrm{F}_{\mathrm{G}}^{*}$ by combining the two forces, buoyancy and gravity, to get:

$$
\mathrm{F}_{\mathrm{G}}^{*}=\frac{\pi \mathrm{d}_{\mathrm{p}}^{3}}{6} \mathrm{~g} \Delta \rho
$$

where $\Delta \rho$ is the difference between the particle density and the fluid density.

In addition, the Reynolds number $\left(R_{e}\right)$ and the Froud number $\left(F_{r}\right)$ are defined by

$$
\begin{aligned}
& R_{\mathrm{e}}=\frac{\mathrm{d}_{\mathrm{p}}\langle\mathrm{v}\rangle \Delta \rho}{\mathrm{n}}=\frac{\text { Inertial forces }}{\text { viscous forces }} \\
& \mathrm{F}_{\mathrm{r}}=\frac{\langle\mathrm{v}\rangle^{2}}{\mathrm{gd}_{\mathrm{p}}}=\frac{\text { Inertial forces }}{\text { gravitational forces }}
\end{aligned}
$$

If the fluid within a tube is in horizontal motion along the $x$-axis, and the Reynolds number is less than one, a parabolic laminar flow pattern will develop neglecting end effects ${ }^{3.4}$ with an average flow velocity given by $\langle v\rangle$. The spherical particle will follow the flow field with a velocity, $v_{p}$. This velocity will be less than or equal to the average flow velocity.

According to Stokes law, for $R_{\mathbf{e}}<1$, the drag coefficient is give by

$$
C_{D}=\frac{24}{R_{e}}
$$

If we look again in terms of forces, we can use dimensional analysis ${ }^{4}$ to obtain a dimensionless group from the ratio of $F_{G}$. to $F_{D}$. By substituting equations 5 and 7 into this ratio, we obtain the following equation

$$
\frac{\mathrm{F}_{\mathrm{G}}^{*}}{\mathrm{~F}_{\mathrm{D}}}=\frac{\mathrm{d}_{\mathrm{p}}^{2} \Delta \rho \mathrm{g}}{18 \eta\langle\mathrm{v}\rangle}=\frac{\mathrm{R}_{\mathrm{e}}}{18 \mathrm{~F}_{\mathrm{r}}}=\frac{\text { gravitational forces }}{\text { viscous forces }}
$$

where $\eta$ is the fluid viscosity.

As can be seen from equation 8 , we have a dimensionless group of variables that can be viewed as the ratio of the Reynolds number to the Froud number, or as the ratio of gravitational forces to viscous forces. By looking at equation 8 more closely, we can assess which variables are going to be important in influencing the relative magnitudes of these two forces. For example, an increase in the size and density of a particle will tend to increase the ratio of these two forces, whereas an increase in viscosity and mean fluid velocity will tend to decrease this ratio. 
Another way to view this is to look again in terms of velocity components. The average velocity is given by

$$
\langle v\rangle=\int_{\mathbf{A}} \mathrm{vdA}
$$

and the Stokes settling velocity of the particle is given by

$$
v_{s}=\frac{g \Delta \rho d_{p}^{2}}{18 \eta}
$$

If we take the ratio of the average flow velocity to the settling velocity we obtain

$$
\frac{\mathrm{v}_{\mathrm{s}}}{\langle\mathrm{v}\rangle}=\frac{\mathrm{g} \Delta \rho \mathrm{d}_{\mathrm{p}}^{2}}{18\langle\mathrm{v}\rangle \eta}=\frac{\text { settling velocity }}{\text { flow velocity }}
$$

this is the same dimensionless ratio as mentioned earlier.

There are many underlying assumptions of the particle in a circular tube model that must be considered when drawing conclusions regarding particles traveling through the small intestine. ${ }^{2}$

a. Spherical particles (no lift forces)

b. Low particle density

c. No electrostatic charges are present on the surface of particles

d. Smooth inner radius (neglects the effects of villi and microvilli along the GI tract)

e. Single horizontal tube (neglects the convoluted nature of the small intestine)

f. The volume and viscosity of the fluid entering the tube is the same as the volume and viscosity exiting the tube (neglects fluid absorption and secretion along the small intestine which can contribute to the change in viscosity)

g. A constant flow velocity (neglects the intermittent motor activity along the small intestine which can lead to fluctuations in flow velocity)

h. Reynolds numbers are less than one, so Stokes region applies

Viewing the physical variables that influence gastric emptying and intestinal transit from equation 8 or 11, although simple and approximate, are qualitatively useful. The in vivo results that were obtained by Amidon et al., ${ }^{5-7}$ Williams et al., ${ }^{8}$ and Meyers et al. ${ }^{9}$ suggest that the dimensionless ratio, $R_{e} / F_{r}$, is a good correlating variable and can provide insight into the importance of gravity on gastric emptying and intestinal transit.

Other physical factors that can specifically influence the rate of gastric emptying include volume and calories, exercise, density and size, temperature, osmolality, and $\mathrm{pH}$.

\section{Volume/Calories}

Two important factors that alter gastric emptying are the volume and caloric content of the meal. In the fasted state, a noncaloric larger volume will empty at a faster rate than a smaller volume under the same physiologic conditions. ${ }^{10}$ When a caloric meal is introduced, the duodenum appears to act through a negative feedback mechanism to allow only a fixed rate of calories to be delivered from the stomach regardless of either the type of calories or the caloric density ingested. ${ }^{11}$ The initial period of caloric delivery, 0-30 minutes, is not characterized by the same linear relationship, but by a faster rate, which is the result of increased intragastric volume occurring before the feedback inhibition is established. ${ }^{12}$

\section{Exercise}

Exercise can play an important role in drug disposition. An increasing number of individuals are taking drugs for a variety of physical and psychologic conditions while concurrently engaging in a specific athletic regimen. This exercise program may have potentially adverse repercussions on the effectiveness of the drug therapy through alterations in GI emptying, GI transit, and GI secretions. For instance, workloads in which $70 \%$ of the maximum oxygen uptake is achieved tend to decrease gastric emptying of liquids and solids, whereas lighter workloads have an opposite or negligible effect. ${ }^{13,14}$

\section{Size and Density}

Due to the unique motility and structure of the stomach, the pylorus acts as a sieve allowing only particles of a certain size range to be emptied with a meal while larger particles must wait for the stronger phase III contractions at the end of the meal to empty. Investigators have shown that this particle size cutoff is about $3.0 \mathrm{~mm}$ in both dogs and humans and is also dependent on the particle density. ${ }^{6,7}$

\section{Temperature}

According to a recent study in humans in which hot, warm, and cold beverages were administered to human subjects directly into the stomach, no significant alteration in GI emptying was observed..$^{15}$ This finding is supported by other evidence in the literature for both dogs and humans. ${ }^{16,17}$ 


\section{Viscosity}

Meal viscosity affects gastric emptying with low viscosity liquids being emptied at a faster rate than higher viscosity liquids. ${ }^{18}$

\section{Osmolality}

Osmolality has been shown to affect GI emptying with noncaloric isotonic meals emptying faster than either hypertonic or hypotonic meals. ${ }^{19}$ The receptor for this regulation appears to be present in the duodenum. ${ }^{20}$

Fluid flow has a major influence on intestinal transit and digestion of food particles in the small intestine. A study that illustrates this effect used radiolabeled liver particles of $0.2,0.5,1.0$, and $2.0 \mathrm{~mm}$ size ranges during intestinal perfusion of a dog in the fed state. ${ }^{8}$ Small particles, less than $0.7 \mathrm{~mm}$, were shown to move with the fluid along the GI tract. At slower flow rates, less than $2 \mathrm{~mL} / \mathrm{min}$, both large and small particles tended to move with the fluid. As the flow rate was increased above $2 \mathrm{~mL} / \mathrm{min}$, the larger particles moved through the intestine at significantly slower speeds $(20-25 \%)$ than the smaller particles. These results are consistent with other sources. ${ }^{9}$ As fluid flow was increased above $2 \mathrm{~mL} /$ min, there was an increase in transit of all particle sizes, but the relative transit rates between the smaller and larger particles remained the same. All particles less than $0.7 \mathrm{~mm}$ traveled through the intestine at roughly the same rate as the fluid while the 1 and $2 \mathrm{~mm}$ particles traveled slower, but not significantly different from each other. This suggests a region between 0.5 to $1.0 \mathrm{~mm}$ during which particle size could have marked effects on transit.

Differences between median transit times of PEG, $0.5 \mathrm{~mm}$ particles, and $2 \mathrm{~mm}$ particles become insignificant after a $200 \mathrm{~g}$ steak meal as compared with the significant differences shown during perfusions. ${ }^{8}$ The author points to the increase in viscosity of the chyme as a possible reason for this observation. This hypothesis is supported by a previously mentioned in vitro study ${ }^{2}$ in which it was shown that an increase in viscosity of the flowing medium through a $2 \mathrm{~m}$ tygon tube to a value greater than twice that of water yielded nearly identical transit rates between particles with diameters and densities ranging from $45 \mu \mathrm{m}$ to $340 \mu \mathrm{m}$ and $1.1 \mathrm{~g} / \mathrm{cm}^{3}$ to $6.9 \mathrm{~g} / \mathrm{cm}^{3}$, respectively.

\section{Physiologic Factors}

Gastric emptying and intestinal transit are dependent on a number of physiologic factors including gastric and intestinal motility, splanchnic blood flow, $\mathrm{pH}$, body position, fluid intake, and electrolyte balance. ${ }^{21-24}$ In the normal fasted state the GI motility is cyclical and periodic in nature which leads to a highly variable gastric emptying in animals and humans. The gastric emptying rate is dependent upon the motility phase, i.e., gastric emptying is slowest in phase I (quiescent phase), somewhat faster in phase II (intermittent contractile activity), and fastest in phase III (strong contractile activity). This periodic contractile activity reoccurs about every two hours and propagates from the stomach down the intestine to the ileocecal region. Thus, gastric emptying may be altered by variations in the overall cycle length as well as the length of the individual phase components.

In the fed state, the gastric emptying pattern is significantly different from the pattern in the fasted state. Gastric emptying is principally dependent upon the number of calories delivered to the small intestine via intragastic contractions. These contractions of the gastrointestinal smooth muscle are triggered by depolarization of the resting transmembrane depolarization potential maintained by each cell. ${ }^{25}$ This depolarization is reported to be brought about and controlled by stimuli which raise transmembrane potential such as acetylcholine. During space flight, the relative absence of gravity is reported to cause cardiovascular changes which could lead to alterations in splanchnic blood flow, and a high incidence of motion sickness resulting from excessive vestibular stimulation. This excessive stimulation leads to disturbances in acid secretions altering the normal electrolyte balance. This in turn changes the contractibility of muscles, which alters the rhythmic contractions of the stomach and intestine and ultimately changes the normal gastric emptying and intestinal transit pattern.

\section{IMPLICATIONS FOR DRUG ABSORPTION (GRAVITY ENVIRONMENT)}

\section{Variability of Gastric Emptying}

These effects on gastric emptying and GI transit may have definite implications for oral drug absorption. Variability in gastric emptying can lead to variability in drug and/or metabolite levels of drugs. Examples include diphenhydramine and aspirin which require rapid absorption to treat acute conditions and propranolol and verapamil which have substantial first-pass metabolism. ${ }^{26-28}$ Also, variabilities associated with emptying have been suggested as a plausible explanation for the unusual plasma level time phenomenon seen with cimetidine. ${ }^{29}$ 


\section{Variability of Intestinal Transit}

Variabilities in intestinal transit rate can affect the therapeutic effectiveness of oral drugs. Abnormal rates in GI transit can disrupt the optimum contact time between the drug and its absorptive site along the intestine and can yield highly variable plasma levels. This has been shown for $\beta$-lactam drugs with nonlinear absorption where the extent of absorption was modelled using a stochastic variability in small intestine transit time.

\section{Implications for Drug Absorption (Microgravity Environment)}

Fed State Gastric Size Discrimination. Microgravity may play a vital role in altering the particle size discrimination of the stomach. In space, the absence of gravity would have profound effects on the influences of size and density on GI emptying. Little dependence on density would be expected since there is no gravity to distinguish between a high- and a low-density particle. However, the effects of particle size on emptying are less easy to hypothesize. Based on preflight and inflight salivary monitoring of drug levels, acetaminophen appears to be emptied in an erratic nonreproducible fashion. ${ }^{31}$ GI Emptying might be envisioned as a probability occurrence limited only to the random chance that a particle in the gut will pass through the pyloric opening. Moreover, the particle is not restricted by gravity to the lower pyloric region of the stomach once it passes the gastroesophageal sphincter, but moves throughout all areas of the stomach from one inelastic collision to the next. Therefore, emptying would appear to be more dependent on the kinetic energy of a particle - specifically its velocity resulting from pressure effects generated by the stomach, particle/particle, and particle/gastric wall collisions - than on particle size.

Intestinal Transit Rate. By looking at the fluid mechanic aspect of intestinal flow as discussed previously, in the presence of a microgravity environment (equation 8), two contributing factors to the longer residence time of the particle are currently removed. Based on equations 1 through 3 , the drag force would remain significant, hence, the particle would move with the fluid stream. Therefore, it might be expected that an exposure to microgravity, assuming velocity and average flow velocity are not significantly altered, would yield faster transit times in many instances; especially with the smaller particles of a meal. These faster transit rates can have adverse effects on the proper absorption of nutrients as well as drugs.

Conclusions. A basic understanding of the forces acting upon a particle traveling along the GI tract is essential to determining the important factors that can influence the movement of a particle. Specifically, the most significant dimensionless group influencing relative particle motion is the ratio of the gravitational force to the viscous force on the particle.

In the fasted state, the rate of gastric emptying and intestinal transit depend highly on the motility phase of the stomach. In the fed state, however, gastric emptying depends on many factors, e.g., calories, particle size, and viscosity, whereas intestinal transit is less influenced by particle size, i.e., both the particle and the fluid move at a similar rate.

The drug and nutritional implications are fourfold: 1) more variable gastric emptying occurs in the fasted state than in the fed state in a gravity environment. This variability may not be as well defined in a microgravity situation; 2) alteration in particle size emptying in microgravity; 3) intestinal adaptation and alteration in transit rate in direct and/or indirect response to zero gravity; and 4) coupling of physiologic changes with alterations in physical properties of gastric contents brought about by exposure to a microgravity environment leading to changes in emptying and transit in both the fasted and fed state.

\section{REFERENCES}

1. Cintron NM, Putcha L, Chen YM, Vanderploeg JM: Inflight salivary pharmacokinetics of scopolamine and dextroamphetamine. Results of the Life Sciences DSOs Conducted Aboard the Space Shuttle 1981-1986. May 1987;NASA:25-29.

2. Najib N, Mansour AR, Amidon GL: Gastrointestinal transit time: An in vitro study of parameters controlling the mean relative residence times of particles in a laminar fluid flowing in a horizontal tube. Chem Engl J 1988;37:B39-B46.

3. Denn MM: Process Fluid Mechanics. Amundson NR (ed.). New Jersey: PrenticeHall, 1980;52-65.

4. Brodkey RS: The Phenomena of Fluid Motions. Hoelscher H (ed.). Columbus, Ohio: Addison-Wesley, 1967;108-109, 129-131, 621-623.

5. Amidon GL: Fluid mechanics and intestinal transit (letter to the editor). Gastroenterology 1985;88:858.

6. Meyer JH, Dressman J, Fink A, Amidon G: Effect of size and density on canine gastric emptying of nondigestible solids. Gastroenterology 1985;89:805-813.

7. Meyer JH, Elashoff J, Porter-Fink V, Dressman J, Amidon GL: Human postprandial gastric emptying of 1-3 millimeter spheres. Gastroenterology 1988;94:315-325.

8. Williams NS, Meyer JH, Jehn D, Miller J, Fink AS: Canine intestinal transit and digestion of radiolabeled liver particles. Gastroenterology 1984;86:1451-1459.

9. Meyer JH, Thomson JB, Cohen MB, Shadchehr A, Mandiola SA: Sieving of solid food by the canine stomach and sieving after gastric surgery. Gastroenterology 1979;76:804-813. 
10. Hunt JN, Spurrell WR: The pattern of emptying of the human stomach. J Physiol 1951;113:157-168.

11. Brener W, Hendrix TR, McHugh PR: Regulation of the gastric emptying of glucose. Gastroenterology 1983;85:76-82.

12. Hunt JN, Smith JL, Jiang CL: Effect of the meal volume and energy density on the gastric emptying of carbohydrates. Gastroenterology 1985;89:1326-1330.

13. Ramsbottom N, Hunt JN: Effect of exercise on gastric emptying and gastric secretion. Digestion 1974;10:1-8.

14. Kasabach $\mathrm{HH}$ : Effect of ingestion of water and of dextrose solution on the emptying time of the normal stomach. Arch Intern Med 1931;48:1237-1240.

15. McArthur KE, Feldman M: Gastric acid secretion, gastrin release, and gastric emptying in humans as affected by liquid meal temperature. Am J Clin Nutr 1989;49:51-54.

16. Teeter BC, Bass P: Gastric emptying of liquid test meals of various temperatures in the dog. Exp Biol Med 1982;169:527-531.

17. Bateman DN: Effects of meal temperature and volume on the emptying of liquid from the human stomach. $J$ Physiol 1982;331:461-467.

18. Prove J, Ehrlein H-J: Motor function of gastric antrum and pylorus for evacuation of low and high viscosity meals in dogs. Gut 1982;23:150-156.

19. Hunt JN: The duodenal regulation of gastric emptying. Gastroenterology 1963;45:149-156.

20. Meeroff JC, Go VLW, Phillips SF: Control of gastric emptying by osmolality of duodenal contents in man. Gastroenterology 1975;68:1144-1151.

21. Winne D: Influence of blood flow on intestinal absorption of xenobiotics. Pharmacology 1980;21:1-15.
22. Levine RR: Factors affecting gastrointestinal absorption of drugs. Am J Dig Dis 1970;15:172-188.

23. Nimmo $W$, Prescott $L$ : The influence of posture on paracetamol absorption. Br J Clin Pharmacol 1978;5:157-168.

24. Graybiel A: The prevention of motion sickness in orbital flight, in: Life Sciences and Space Research XIV, Berlin: AkademieVerlag, 1976;110-118.

25. Kumar D, Crustavsson D (eds.): Gastrointestinal Motility. New York: John Wiley and Sons, 1988;163-175.

26. Wagner JG: Propranolol: Michaelis-menten parameters and the effect of input rate on bioavailability. Clin Pharm Ther 1985;37:481-487.

27. Wagner JG: Comparison of nonlinear pharmacokinetic parameters estimated from the sinusoidal perfusion and venous equilibration models. Biopharm Drug Dispos 1985;6:23-31.

28. Rose S: The effect of input rate on the plasma disposition of propranolol in the dog (thesis). The University of Michigan, 1990.

29. Oberle RL, Amidon GL: Pharmacometrics: The influence of variable gastric emptying and intestinal transit rates on the plasma level curve of cimetidine; an exploration for the double peak phenomenon. J Pharmacokinet Biopharm 1987;15:529-544.

30. Sinko PJ, Amidon GL: Characterization of the oral absorption of $\beta$-lactam antibiotics. I. Cephalosporins: Determination of intrinsic membrane absorption parameters in the rat intestine in situ. Pharm Res 1988;10:645-650.

31. Cintron NM, Putcha L, Vanderploeg JM: Inflight pharmacokinetics of acetaminophen in saliva. Results of the Life Sciences DSOs Conducted Aboard the Space Shuttle 1981-1986. May 1987;NA 